\title{
Generation of Mice Deficient for Macrophage Galactose- and $N$-Acetylgalactosamine-Specific Lectin: Limited Role in Lymphoid and Erythroid Homeostasis and Evidence for Multiple Lectins
}

\author{
Thandi M. Onami, ${ }^{1,2}$ Meei-Yun Lin, ${ }^{1,2}$ Dawne M. Page, ${ }^{1,2}$ Shirley A. Reynolds, ${ }^{1,2}$ \\ Carol D. Katayama, ${ }^{1,2}$ Jamey D. Marth, ${ }^{3,4}$ Tatsuro Irimura, ${ }^{5}$ Ajit Varki, ${ }^{6,7}$ \\ Nissi Varki, ${ }^{6,7}$ and Stephen M. Hedrick ${ }^{1,2 *}$ \\ Molecular Biology Section, Division of Biology, ${ }^{1}$ Cancer Center, ${ }^{2}$ Howard Hughes Medical Institute,${ }^{3}$ Department of \\ Cellular and Molecular Medicine, ${ }^{4}$ Department of Medicine, ${ }^{6}$ and Glycobiology Research and Training Center, ${ }^{7}$ University \\ of California, San Diego, La Jolla, California 92093, and Laboratory of Cancer Biology, University of \\ Tokyo, Tokyo 113-8657, Japan ${ }^{5}$
}

Received 21 March 2002/Accepted 2 April 2002

\begin{abstract}
Macrophage receptors function in pattern recognition for the induction of innate immunity, in cellular communication to mediate the regulation of adaptive immune responses, and in the clearance of some glycosylated cells or glycoproteins from the circulation. They also function in homeostasis by initiating the engulfment of apoptotic cells. Evidence has suggested that macrophage receptors function to recognize cells that are destined for programmed cell death but not yet overtly apoptotic. We have examined the function of a macrophage receptor specific for unsialylated glycoproteins, known as the mouse macrophage galactose- and $N$-acetylgalactosamine-specific lectin (mMGL) (Ii et al., J. Biol. Chem. 265:11295-11298, 1990; Sato et al., J. Biochem. [Tokyo] 111:331-336, 1992; Yamamoto et al., Biochemistry 33:8159-8166, 1994). With targeted disruption, we tested whether mMGL is necessary for macrophage function, controlled thymic development, the loss of activated CD8 T cells, and the turnover of red blood cells. Evidence indicates that mMGL may play a nonessential role in several of these macrophage functions. Experiments are presented that indicate the existence of another galactose- and $N$-acetylgalactosamine-recognizing lectin distinct from mMGL. This may explain the absence of a strong phenotype in mMGL-deficient mice.
\end{abstract}

Differential sialylation is a general property of hematopoietic cell development, including lymphocytes and erythrocytes. For example, at a distinct stage in thymic development, several abundant cell surface glycoproteins, such as CD8, CD43, and CD45, are undersialylated, thus exposing terminal O-linked Galß1-3GalNAc, a ligand for the lectin peanut agglutinin (PNA). T-cell precursors migrate to the thymus as $\mathrm{PNA}^{\text {lo }}$ $\mathrm{CD}^{-} \mathrm{CD}^{-}$(double negative [DN]) cells $(24,38)$ and make the transition to $\mathrm{PNA}^{\text {hi }}$ cortical $\mathrm{CD} 4^{+} \mathrm{CD} 8^{+}$(double positive [DP]) cells $(5,18,19,25-27)$. Upon differentiation, the DP thymocytes progress to $\mathrm{CD} 4$ or CD8 lineage cells, migrate to the medulla, and once again become $\mathrm{PNA}^{\mathrm{lo}}$.

The extent of terminal Gal $\beta 1-3$ GalNAc exposure inversely correlates with the expression of ST3Gal-I, a Galß1-3GalNAcspecific $\alpha 2,3$ sialyltransferase that is expressed primarily in medullary but not cortical thymocytes $(3,10)$. Upon expression of ST3Gal-I, Galß1-3GalNAc becomes sialylated, preventing PNA binding on these cells (23). Additionally, T cells become increasingly sialylated on N-linked Gal $\beta 1-4 G l c N A c$ as they transit from DP to single-positive (SP) cells (36). This latter sialylation is thought to involve multiple sialyltransferases ( 7 , 35).

Naive $\mathrm{PNA}^{\text {lo }} \mathrm{T}$ cells once again become $\mathrm{PNA}^{\text {hi }}$ upon mito-

\footnotetext{
* Corresponding author. Mailing address: Department of Biology, University of California, San Diego, La Jolla, CA 92093-0687. Phone: (858) 534-6269. Fax: (858) 534-5611. E-mail: shedrick@ucsd.edu.
}

gen- or antigen-mediated activation $(8,30)$. Effector $\mathrm{T}$ cells reveal terminal Galß1-3GalNAc, possibly through the action of endogenous sialidases (8). The deletion of ST3Gal-I causes all $\mathrm{T}$ cells to remain PNA positive and thus undersialylated on several O-linked glycoproteins. The phenotypic effect of an ST3Gal-I deficiency was a loss of CD8 T cells in the peripheral lymphoid organs via apoptosis (23). The conclusion from these studies was that regulated sialylation plays a role in the homeostatic maintenance of CD8 T cells.

Mouse macrophage galactose- and $\mathrm{N}$-acetylgalactosaminespecific C-type lectin (mMGL) is a macrophage-specific, calcium-dependent C-type lectin whose carbohydrate recognition domain has been shown to have high affinity for glycoproteins bearing terminal galactose (Gal) and $N$-acetylgalactosamine (GalNAc) sugars (39). This lectin is also known as the macrophage asialoglycoprotein-binding protein by the group that cloned the gene from the rat (12). mMGL binding is inhibited by sialylation (39). It is expressed on a subpopulation of macrophages present in most tissues (20), and it has been implicated in macrophage tumoricidal ability, phagocytosis, and endocytosis $(11,15)$. mMGL expression is also upregulated during cardiac allograft rejection (28). Currently, the physiological role of mMGL remains uncertain.

Based on the expression of mMGL in the thymus and peripheral lymphoid organs and the existence of cells bearing potential ligands for this terminal Gal/GalNAc-specific lectin, we examined the role of mMGL-bearing cells in the regulation 
and turnover of lymphocytes during development and activation. In this report we describe the generation of mice that are deficient for mMGL. An analysis of these mice revealed that the loss of mMGL did not affect lymphoid differentiation or immune function. Further, red blood cell turnover and life span are minimally affected. We also show that mMGL is not the only Gal/GalNAc-specific lectin expressed by mouse macrophages, and its inhibition does not substantially complement the ST3Gal-I deficiency. We conclude from these studies that mMGL plays a role in T-cell and erythrocyte homeostasis but that there are other macrophage lectins with redundant or overlapping functions.

\section{MATERIALS AND METHODS}

Flow cytometry. Cells were labeled with the following monoclonal antibodies and reagents: anti-CD4-phycoerythrin (PE), anti-CD8-TC (CalTag), anti-CD5fluorescein isothiocyanate (FITC), anti-CD3-FITC, anti-heat-stable antigen-biotin, anti-CD44-PE, anti-CD25-biotin or -FITC, anti-CD62L-PE (all from Pharmingen), PNA-FITC, and Erythrina cristigalli agglutinin-biotin (Sigma Chemical Co., St. Louis, Mo.). Apoptotic cells were detected with annexin V-FITC (CalTag), or terminal deoxynucleotidyltransferase-mediated dUTP-biotin nick-end labeling (Boehringer Mannheim). Samples were analyzed with a FACSCAN or FACSCalibur (Becton Dickinson) and CellQuest software.

Genomic mapping and knockout construct. Exons 2 and 3 encoding the translational start site, cytoplasmic domain, and the transmembrane domain of mMGL were mapped from genomic clones isolated from a strain 129SvJ library (Stratagene, La Jolla, Calif.). The probe used was a mouse full-length mMGL cDNA isolated from a subtractive hybridization experiment and identified by sequence as previously published (3). These exons were replaced with the pPGKneobpA cassette (32), resulting in a targeting construct consisting of a $1.5-\mathrm{kb}$ and a 4-kb arm to mediate homologous recombination. The construct was transfected into R1 embryonic stem (ES) cells (129 strain), and 700 G418-resistant colonies were screened for homologous recombinants. One clone, H1, was identified and injected into C57BL/6 blastocysts. Male mice obviously chimeric by coat color were crossed to $\mathrm{C} 57 \mathrm{BL} / 6 \mathrm{~J}$ females to obtain $\mathrm{F}_{1}$ heterozygotes, which were then incrossed to obtain $\mathrm{F}_{2}$ mice homozygous for the deficient $m M G L$ allele.

Immunohistochemistry. Tissues were collected from mice, frozen in OCT (VWR Scientific), and kept at $-70^{\circ} \mathrm{C}$ until sectioned on a cryostat. Sections were acetone fixed, washed in Tris-buffered saline, and blocked with $2 \%$ bovine serum albumin. Biotinylated and unbiotinylated polyacrylamide arrays carrying multiple copies of Galß1-3GalNAc and Gal $\alpha 1-3$ GalNAc were purchased from Glycotech (Rockville, Md.). Antifreeze glycoprotein (AFGP; Sigma Chemical Company) was biotinylated with a biotinylation kit (Sigma Chemical Company). Rat anti-mouse mMGL monoclonal antibody LOM-14 (13) was prepared as previously described. Biotinylated anti-Mac-1 and FITC-conjugated anti-Mac-1 were purchased from BD Pharmingen.

Sections were incubated with primary reagents at room temperature for at least $1 \mathrm{~h}$, washed, and incubated for $30 \mathrm{~min}$ with secondary goat anti-rat alkaline phosphatase (Jackson Immunochemicals). Sections were then developed with alkaline phosphatase substrate and Vector Blue, and nuclei were counterstained with Nuclear Fast Red (Vector Labs, Burlingame, Calif.). Alternatively, primary biotinylated reagents were developed with fluorescently tagged streptavidin or streptavidin-alkaline phosphatase.

Fibroblasts expressing mMGL. Full-length mMGL cDNA was subcloned into pcDNA3 (Invitrogen, Carlsbad, Calif.) and transfected into 3T6 fibroblasts with Polybrene as described before (21). G418-resistant clones were tested for mMGL expression with flow cytometry and Northern analysis. Positive clones were subcloned by limiting dilution. Transfected fibroblasts were seeded into 24 -well plates at $10^{5}$ cells/well overnight.

Peritoneal macrophages. Littermates aged 4 to 8 months were challenged with $1 \mathrm{ml}$ of $3 \%$ thioglycollate intraperitoneally, and 4 days later peritoneal cells were isolated by lavage with $10 \mathrm{ml}$ of cold Hanks' balanced salt solution. Cells were washed, counted, and stained for Mac-1. Cells were resuspended in RPMI medium, seeded with $10^{6}$ Mac- $1^{+}$cells to form monolayers, and allowed to adhere to wells for 2 to $4 \mathrm{~h}$. Nonadherent cells were vigorously washed with medium.

In vitro coculture. Thymocytes were explanted from C57BL/6 mice and cultured as described above. Thymocytes $\left(4 \times 10^{6}\right.$ to $\left.5 \times 10^{6}\right)$ were added to triplicate wells containing monolayers of macrophages or fibroblasts in $1 \mathrm{ml}$ of

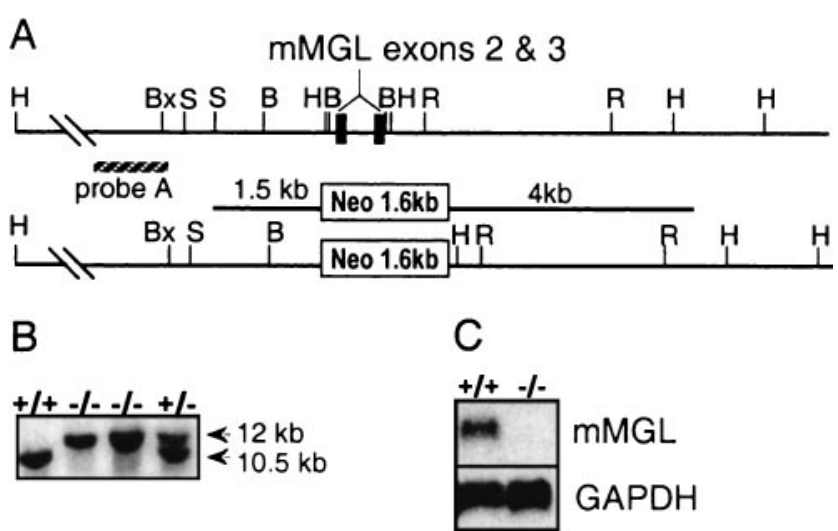

FIG. 1. Targeted deletion of $m M G L$ exons 2 and 3 results in mice deficient for mMGL. (A) Genomic mapping of the mMGL aminoterminal cytoplasmic and transmembrane domains revealed $\mathrm{a} \approx 1.0 \mathrm{-kb}$ HindIII fragment encoding exons 2 and 3 which was targeted for deletion with replacement vector pPGKneo (23). (B) Tail DNA from $\mathrm{F}_{2}$ mice digested with HindIII and probed with an upstream genomic probe revealed an endogenous $10.5-\mathrm{kb}$ fragment and the recombinant $12-\mathrm{kb}$ fragment. (C) Northern analysis of $m M G L$ mRNA in $m M G L^{+/-}$ and $m M G L^{-1-}$ mice. GAPDH, glyceraldehyde-3-phosphate dehydrogenase. H, HindIII; S, SacI; R, Eco RI; Bx, BstXI; B, BamHI; N, NotI.

medium for 16 to $18 \mathrm{~h}$. After vigorous washing, $0.2 \mathrm{ml}$ from each well was stained, counted, and analyzed by flow cytometry to determine live cell recovery. Recovery was calculated by multiplying the fraction of nonapoptotic cells (annexin $\mathrm{V}^{-}$) of a specific subset by the total number of cells recovered.

CTL assay. Cytotoxic T lymphocyte (CTL) assays were performed as previously described (23). Briefly, mice were injected with $2 \times 10^{6}$ P815 tumor cells intraperitoneally. On day 10 , spleens and mesenteric lymph nodes were isolated from mice, and lymphocytes were counted. Lymphocytes were added to 96-well V-bottomed plates containing ${ }^{51} \mathrm{Cr}$-labeled specific target cells (P815) or nonspecific targets (EL-4) at various effector-to-target cell ratios. Cells were incubated for $4 \mathrm{~h}$ with targets, and ${ }^{51} \mathrm{Cr}$ release was measured.

Determination of erythrocyte life span. To determine the life span of erythrocytes, $m M G L$-deficient mice and control littermates were biotinylated in vivo with $N$-hydroxysuccinimido-biotin (NHS-biotin) (Sigma, St. Louis, Mo.) as described previously (1). Briefly, NHS-biotin was dissolved in dimethyl sulfoxide (Aldrich, Milwaukee, Wis.) and diluted 1:10 with phosphate-buffered saline (PBS) (Sigma) to a final concentration of $4 \mathrm{mg} / \mathrm{ml}$, and $150 \mu \mathrm{l}$ of the final solution was injected into the tail vein. The injection was repeated $1 \mathrm{~h}$ later. Blood from biotinylated mice was taken at 1-week intervals, and $50 \mu \mathrm{l}$ of blood was washed with $2 \mathrm{ml}$ of PBS supplemented with $2 \%$ fetal calf serum and $1 \mathrm{mM}$ EDTA to remove biotinylated plasma proteins. Triplicate samples containing 15 $\mu \mathrm{l}$ of blood cells were stained with $0.5 \mu \mathrm{g}$ of streptavidin-PerCP (Pharmingen, San Diego, Calif.) at room temperature for $30 \mathrm{~min}$. The percentage of labeled erythrocytes was determined by flow cytometry, and the disappearance of the cells over time was used to determine the erythrocyte life span for each mouse.

\section{RESULTS}

Generation of $\mathbf{m M G L ^ { - 1 - }}$ mice. To understand the physiological role of mMGL in vivo, we targeted mMGL for germ line deletion in mice. Since mMGL is a type II transmembrane protein, we mapped the $\mathrm{N}$-terminal transmembrane domain and targeted exons 2 and 3 for replacement with a neomycin resistance cassette with the vector pPGKneo (Fig. 1A). Homologous recombinants in ES cells could be identified by a $\approx 12-\mathrm{kb}$ HindIII recombinant fragment, compared to a $\approx 10.5$-kb endogenous fragment when probed with an upstream genomic probe. Of 700 G418-resistant ES clones examined by Southern blot analysis, one clone revealed the expected recombinant allele, and this clone was injected into blastocysts. 

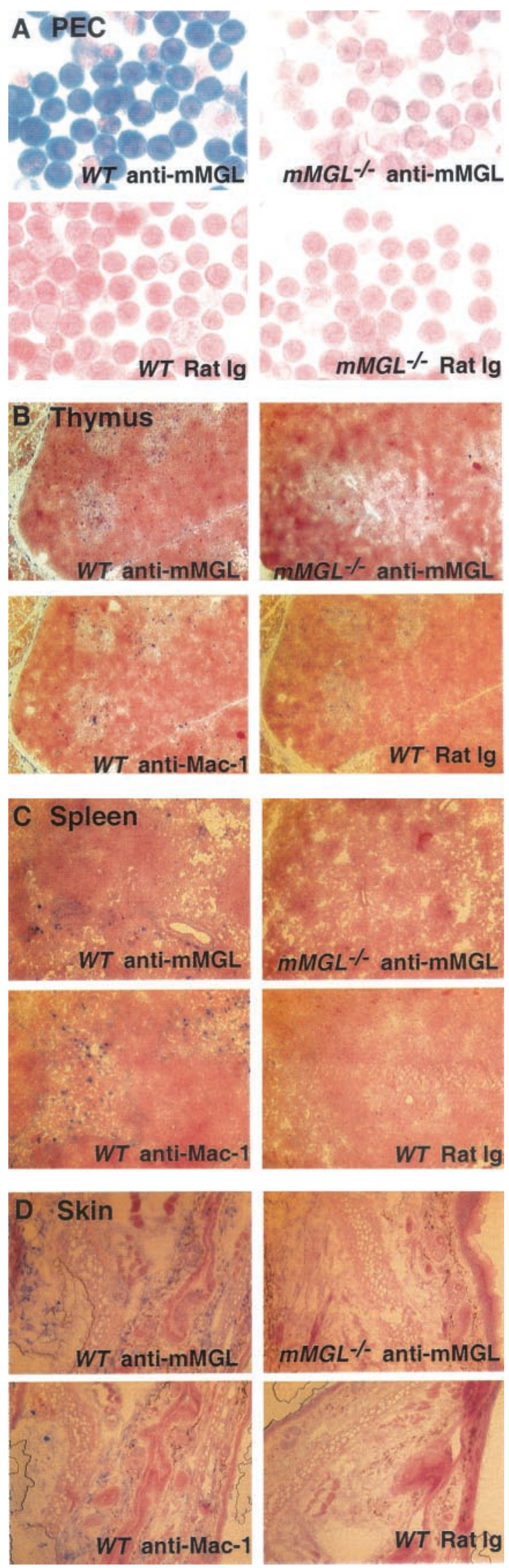
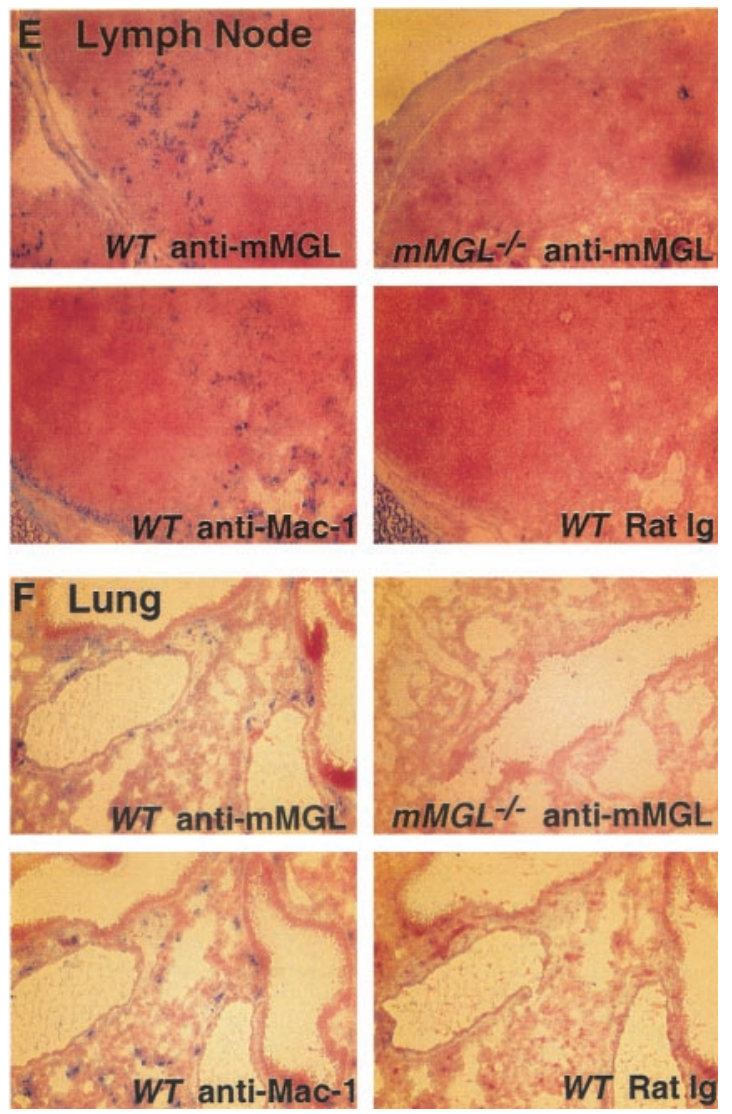

FIG. 2. Localized expression of mMGL in mouse tissues. Tissue sections from $\mathrm{mMGL}^{+/+}$and $\mathrm{mMGL}^{-/-}$mice were stained with rat immunoglobulin (Ig) control or monoclonal anti-mMGL antibodies and compared to Mac-1 staining in each tissue. (A) Cytospin of peritoneal exudate cells. (B) Thymus. Note that cells staining for mMGL in wild-type (WT) mice were seen predominantly in the corticomedullary junction and medulla. (C) Spleen, showing sparse staining of mMGL staining compared to that in skin (D), lymph node (E), and lung $(\mathrm{F})$.

The resulting chimeric mice passed the deficient allele to the first-generation progeny (Fig. 1B). Homozygous deficient mice were deficient for $m M G L$ by Northern (Fig. 1C) as well as by reverse transcription-PCR analysis (data not shown).

mMGL expression. In order to verify the absence of mMGL expression in $m M G L^{-1-}$ mice and further characterize mMGL expression in different tissues, immunohistochemistry was carried out with anti-mMGL monoclonal antibody (LOM14). Figure $2 \mathrm{~A}$ depicts the staining from a cytospin of peritoneal exudate cells. As shown, a large percentage of peritoneal exudate cells from wild-type mice were strongly mMGL positive. The staining of peritoneal exudate cells from $m M G L^{-1-}$ mice was greatly diminished, although, with purified antibody, we did detect residual staining over the rat immunoglobulin background.

We also compared the expression of mMGL to that of Mac-1 in thymus, spleen, lymph node, skin, and lung from wild-type and $m M G L$-deficient mice. In the thymus, we found medullary staining in the wild-type mouse that was again reduced in the thymuses from $m M G L^{-/-}$mice (Fig. 2B). This pattern of medullary staining is intriguing because the thymo- 
A

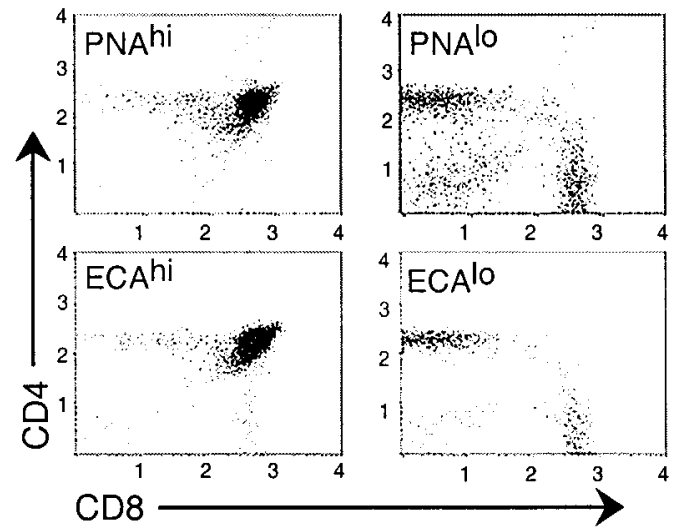

B

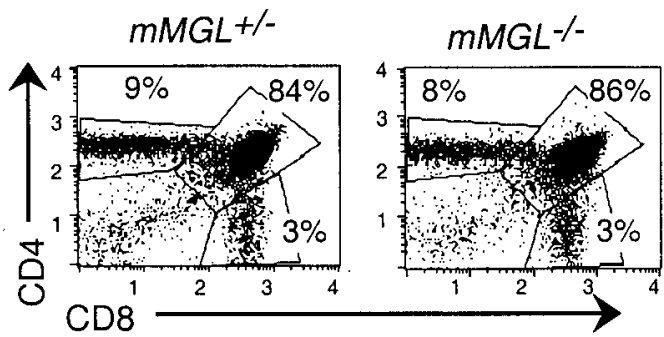

C

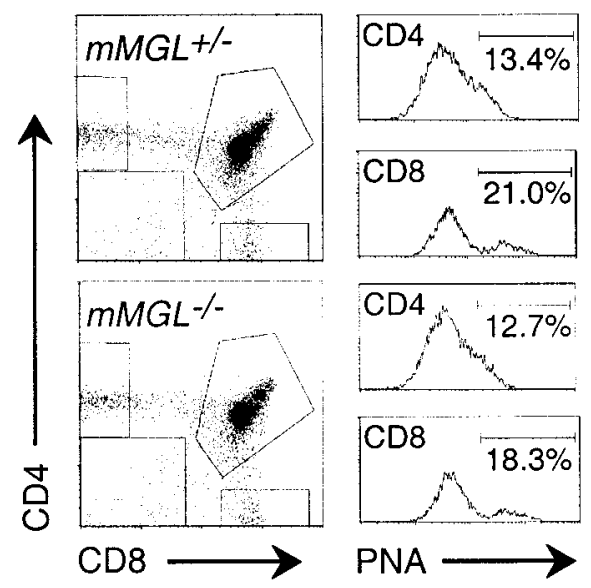

cytes bearing potential ligands for mMGL, namely, unsialylated O-linked Galß1-3GalNAc, are located in the cortex, whereas cells bearing this $\mathrm{Gal} / \mathrm{GalNAc}$ receptor are segregated in the medulla. In the spleen of wild-type mice, we found sparse mMGL staining compared with Mac-1 (Fig. 2C), similar to the staining found in liver (not shown). In contrast, mMGL staining in skin (Fig. 2D) and lymph nodes (Fig. 2E) was at least as frequent as Mac-1 staining. In the skin, abundant staining was seen in the dermis, and in the lymph node there was also abundant staining in the extrafollicular paracortex. These results indicate that mMGL is differentially expressed in Mac- $1^{+}$cells. It is definitely expressed on peritoneal macrophages; however, expression elsewhere is consistent with a subpopulation of macrophages or mature dendritic cells. While the antibody staining revealed by LOM14 is diminished in all tissues from $m M G L^{-1-}$ mice, the possibility remains that there exists another cross-reactive mMGL-like molecule.

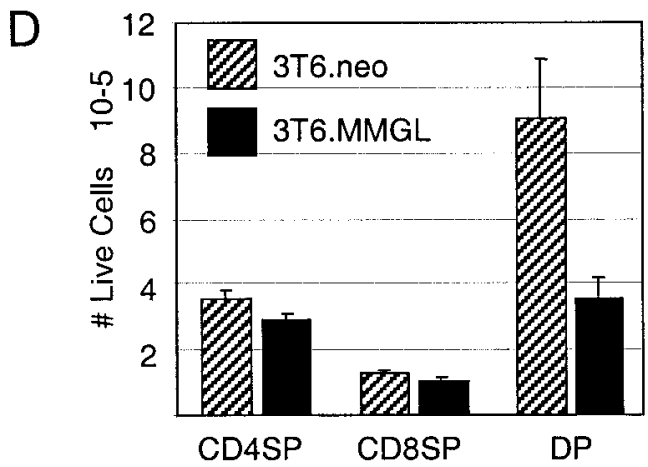

E

Live Cells Recovered $10^{6}$

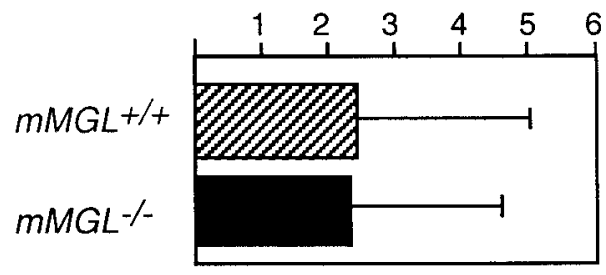

FIG. 3. mMGL null mice show normal T-cell development and homeostasis. (A) Thymocytes were harvested from C57BL/6 mice and stained with anti-CD4-PE, anti-CD8-TC, and either PNA directly conjugated to FITC (top) or biotinylated E. cristigalli agglutinin (ECA) and streptavidin-FITC (bottom). Cells were gated on PNA ${ }^{\text {hi }}$ (left) or PNA $^{\text {lo }}$ (right) to indicate populations with terminal O-linked Galß13 GalNAc or $\mathrm{ECA}^{\text {hi }}$ (left) or $\mathrm{ECA}^{\text {lo }}$ (right) to indicate terminal Nlinked Galß1-4GlcNAc. (B) Thymocytes from $m M G L^{+/-}$and $m M G L^{-1-}$ littermates were stained with anti-CD4 and anti-CD8 and analyzed by flow cytometry. (C) CD4SP (top) and CD8SP (bottom) populations were analyzed for PNA binding between $m M G L^{+/+}$and $m M G L^{-1-}$ mice. (D) Thymocytes were cocultured for $16 \mathrm{~h}$ with $m M G L$ - or mock-transfected 3T6 fibroblasts, and the live cell recovery of different subsets was determined by multiplying cell count by percentage of annexin $\mathrm{V}$ staining on gated populations. (E) Thymocytes from C57BL/6 mice were harvested and cocultured with thioglycollateelicited macrophages from $m M G L^{+/+}$and $m M G L^{-/-}$mice in vitro.

Normal lymphoid development and homeostasis in $\boldsymbol{m} \boldsymbol{M} \boldsymbol{G L}^{-1-}$ mice. We examined $m M G L$-deficient mice for lymphoid development. Our initial hypothesis was that medullary mMGL-expressing macrophages might regulate the developmental transition that occurs from the cortical, undersialylated immature thymocytes to medullary, fully sialylated mature $\mathrm{T}$ cells. This process can be monitored by analyzing thymocytes stained with PNA to detect O-linked terminal Gal-GalNAc and staining for $\mathrm{CD} 4$ and $\mathrm{CD} 8$ to detect the major thymic subpopulations (Fig. 3A). Thymocytes were separately stained with $E$. cristigalli agglutinin, which detects the terminal galactose present on $\mathrm{N}$-linked branched glycoproteins (Fig. 3A). With either of these lectins, we found that undersialylated thymocytes in wild-type mice were mainly $\mathrm{CD} 4^{+} \mathrm{CD} 8^{+}$, the population found in the cortex, whereas the sialylated thymocytes were either $\mathrm{CD}^{+} \mathrm{CD}^{-}(\mathrm{CD} 4 \mathrm{SP})$ or $\mathrm{CD}^{-} \mathrm{CD} 8^{+}$ (CD8SP), the mature populations found in the medulla.

We next sought to determine whether the absence of mMGL-bearing macrophages would result in an altered cellularity within the thymus. Examination of thymuses from $m M G L^{+/-}$and $m M G L^{-/-}$mice revealed that T-cell develop- 
ment appears normal in $m M G L$-deficient mice, with thymocytes having similar subpopulations of $\mathrm{CD}^{+} \mathrm{CD}^{-}$(CD4SP), $\mathrm{CD}^{-} \mathrm{CD}^{+}$(CD8SP), and $\mathrm{CD} 4^{+} \mathrm{CD}^{+}$(DP) cells (Fig. 3B). The number of PNA-high thymocytes in each of the subsets was also determined. The data indicate that there is no significant increase in PNA ${ }^{\text {hi }}$ thymocytes in the mature CD4SP and CD8SP subsets (Fig. 3C). Likewise, development was normal as detected by surface expression of CD3, CD5, and heatstable antigen (data not shown). Further, although variable, the rate of death appeared to be unaltered as measured by staining for apoptotic markers with annexin V, DiOC6, and terminal deoxynucleotidyl transferase-mediated dUTP-biotin nick end labeling (data not shown).

The thymus size of $m M G L$-deficient mice compared to heterozygous littermates seemed slightly increased in mice aged 8 to 16 weeks $\left(9.8[ \pm 4.5] \times 10^{7}\right.$ cells, $n=27[-/-]$ versus 8.4 $[ \pm 3.6] \times 10^{7}$ cells, $\left.n=33[+/+]\right)$, but due to the variability within each group, this difference was not statistically significant $(P>0.05$, Student's $t$ test, unpaired). B-cell expression of B220 was similar among wild-type and mMGL null mice, and B-cell proliferation to anti-immunoglobulin $\mathrm{M}$ and lipopolysaccharide in vitro was normal (data not shown). We conclude from these data that T-cell development is grossly unaltered and that expression of mMGL is not required for normal $\mathrm{T}$ - or B-cell development.

We also studied the function of mMGL with respect to the induction of apoptosis in Gal/GalNAc-exposed CD4 ${ }^{+} \mathrm{CD}^{+}$ thymocytes. In the first set of experiments, we showed that fibroblasts transfected with $m M G L$ would result in cell loss of $\mathrm{CD}^{+} \mathrm{CD}^{+}$(cortical) but not CD4SP or CD8SP (medullary) thymocytes (Fig. 3D).We then sought to determine whether elicited peritoneal macrophages would mediate the loss of thymocytes in an mMGL-dependent manner. Measuring cell recovery, viability, and apoptotic indices, we found that macrophages do have a substantial effect on the recovery of thymocytes, but after extensive investigation, including numerous separate trials, we did not find a significant difference between macrophages from wild-type and $m M G L$-deficient mice (data not shown). Thus, despite the ability of $m M G L$ transfectants to mediate thymocyte cell death in vitro and the demonstrated large amounts of mMGL on peritoneal macrophages (Fig. 2A), there was no indication that mMGL expression was required for thymocyte cell death.

$m M G L$-deficient mice were also tested for T-cell responses by measuring the cytotoxic T-cell response to major histocompatibility-disparate tumor cells. P815 tumor cells were injected intraperitoneally into $m M G L^{+/+}$and $m M G L^{-/-}$mice. On day 10 of the response, CTL specific lysis was measured ex vivo along with the expansion of CD8 T cells. The cytotoxic T-cell responses of $\mathrm{mMGL}^{+/+}$and $\mathrm{mMGL}^{-1-}$ mice were similar (Fig. 4A). Similar expansion of CD8 T cells was also observed (Fig. 4B). Both $m M G L^{+/+}$and $m M G L^{-/-}$T cells exhibited the expression of activation markers, becoming CD44 ${ }^{\mathrm{hi}}, \mathrm{CD}_{62} \mathrm{~L}^{\mathrm{lo}}$, and PNA $^{\text {hi }}$ (data not shown). In addition, we measured the proportion of apoptotic $\mathrm{T}$ cells as measured by annexin $\mathrm{V}$ during the time course of a $\mathrm{T}$-cell response in vivo and again found no significant differences in mice lacking $m M G L$ on day 10 (Fig. 4C), day 12, or day 17 (data not shown). These results indicate that CD8 T-cell responses do not rely on mMGL for activation, effector function, or turnover of activated $\mathrm{T}$ cells.
A

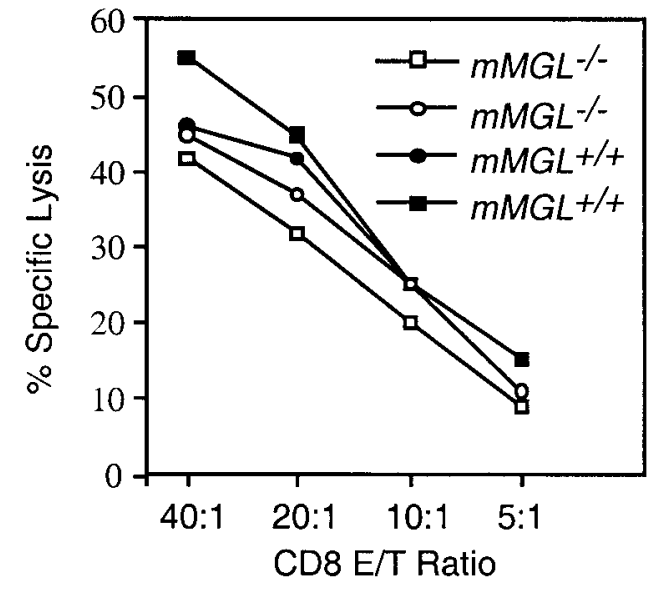

B
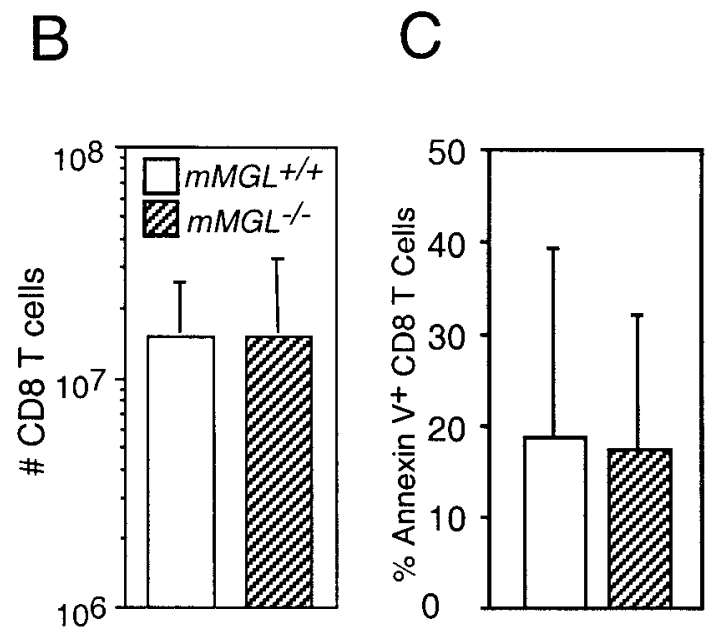

FIG. 4. Allogeneic cytotoxic T-cell response in $\mathrm{mMGL}^{+/+}$and $\mathrm{mMGL}^{-1-}$ mice. (A) P815 targets were cultured with lymphocytes from the spleen, and CTL lysis was determined. $m M G L$-deficient mice showed cytolytic activity similar to that in the wild type. (B) Expansion of CD8 $\mathrm{T}$ cells after the in vivo response was similar between $m M G L^{+/+}$and $m M G L^{-1-}$ mice. (C) Percentage of annexin $\mathrm{V}^{+}$CD8 T cells was similar between $\mathrm{mMGL}^{+/+}$and $\mathrm{mMGL}^{-/-}$mice.

Elevated red blood cell counts but similar life span and turnover. Mice were also subjected to extensive hematology analysis, blood coagulation, and blood chemistry tests. Only measures of erythrocyte content showed a statistically significant deviation from wild-type values. As shown in Table 1, red blood cell counts, hemoglobin, hematocrit, and mean corpuscular volume were all slightly elevated in $m M G L$-deficient mice. This was of potential interest because one model of erythrocyte turnover is that erythrocytes lose sialic acid as a consequence of aging, and it is possible that such aged erythrocytes would be scavenged by macrophages present in the spleen and liver $(9,14,34)$. While the mean differences noted were statistically significant, the $m M G L^{-/-}$values still fell within the normal range for mice. In addition, an analysis of erythrocyte life span in vivo was carried out on $m M G L^{+/+}$and 
TABLE 1. Hematological analysis of $\mathrm{mMGL}^{a}$

\begin{tabular}{|c|c|c|c|c|c|}
\hline $\operatorname{Parameter}^{b}(\mathrm{U})$ & $\mathrm{mMGL}^{+/+}(n=11)$ & $\mathrm{mMGL}^{+/-}(n=10)$ & $\mathrm{mMGL}^{-/-}(n=16)$ & $t$ test & Change (\%) \\
\hline Leukocytes (cells/ $\mu \mathrm{l})$ & 7,772.6 (697.67) & $8,826.0(848.83)$ & $8,830.0(730.08)$ & & \\
\hline Neutrophils $(\%)$ & $8.1(2.02)$ & $11.7(1.55)$ & $9.6(1.24)$ & & \\
\hline Lymphocytes (\%) & $89.1(2.39)$ & $83.2(1.64)$ & $87.1(1.42)$ & & \\
\hline Monocytes (\%) & $0.4(0.18)$ & $1.4(0.24)$ & $0.8(0.21)$ & & \\
\hline Eosinophils (\%) & $0.0(0.00)$ & $0.3(0.12)$ & $0.2(0.08)$ & & \\
\hline Basophils (\%) & $2.4(0.42)$ & $3.3(0.25)$ & $2.2(0.22)$ & & \\
\hline Erythrocytes (m/ml) & $9.8(0.15)$ & $9.9(0.13)$ & $10.2(0.09)$ & 0.044 & +3.5 \\
\hline Hemoglobin (g/liter) & $15.2(0.16)$ & $15.4(0.18)$ & $15.8(0.12)$ & 0.009 & +3.8 \\
\hline Hematocrit $(\%)$ & $47.7(0.52)$ & $48.8(0.54)$ & $50.6(0.41)$ & 0.000 & +6.0 \\
\hline MCV (fl) & $48.6(0.36)$ & $49.3(0.39)$ & $49.8(0.35)$ & 0.032 & +2.4 \\
\hline $\mathrm{MCH}(p g)$ & $15.5(0.10)$ & $15.5(0.12)$ & $15.6(0.10)$ & & \\
\hline MCHC (g/liter) & $31.9(0.10)$ & $31.4(0.13)$ & $31.3(0.10)$ & 0.001 & -1.8 \\
\hline RDW (\%) & $18.7(0.22)$ & $18.3(0.45)$ & $18.3(0.18)$ & & \\
\hline Platelets $\left(10^{3} / \mu \mathrm{l}\right)$ & $820.3(48.4)$ & $861.1(55.8)$ & $928.8(29.7)$ & 0.054 & +13.2 \\
\hline MPV (fl) & $7.0(0.17)$ & $7.1(0.14)$ & $7.0(0.09)$ & & \\
\hline
\end{tabular}

\footnotetext{
${ }^{a}$ Data for each test are listed with the standard error of the mean in parentheses.
}

${ }^{b} \mathrm{MCV}$, mean cell volume; MCH, mean cell hemoglobin; MCHC, mean cell hemoglobin concentration; RDW, red cell distribution width; MPV, mean platelet volume.

$m M G L^{-1-}$ mice. Erythrocyte life spans averaged between 36.67 and 37.27 days, respectively (Fig. 5). We conclude that there are only minor hematological consequences associated with the loss of mMGL.

Complementation of ST3Gal-I deficiency. In mice lacking ST3Gal-I (siat4a), there is a profound loss of CD8 T cells in the periphery (23). In these mice, there is enhanced core 2 glycosylation, revealed by the antibody $1 \mathrm{~B} 11$, and enhanced PNA binding. Moreover, cross-linking with $1 \mathrm{~B} 11$ on CD8 T cells from these mice induced apoptosis (23). We considered the possibility that a lack of ST3Gal-I revealed an unsialylated O-linked Galß1-3GalNAc that could be bound by mMLbearing macrophages in vivo. This, in turn, could cause crosslinking of undersialylated molecules and induce apoptosis. Under such circumstances, a deficiency in $m M G L$ would be predicted to complement the loss of CD8 T cells in ST3Gal-Ideficient mice.

Mice were crossed to produce ST3Gal-I+- controls and

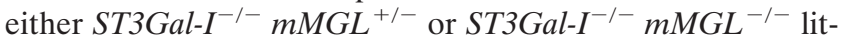
termates (Fig. 6). The number of CD8 T cells was enumerated in the peripheral blood at 3 to 6 weeks of age. In addition, the data were segregated for males and females. As shown in Fig. 6 , there were approximately $11 \%$ CD8 T cells at all ages in ST3Gal- $I^{+/-}$mice, whereas the percentage of CD8 T cells in ST3Gal-I ${ }^{-1-}$ mice varied between 1 and $5 \%$. The absence of $m M G L$ did not complement the level of CD8 T cells to wildtype levels, but there was a trend toward increased T-cell numbers at all ages in double null mice. The difference be-

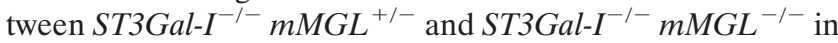
females reached statistical significance at weeks 3 and $5(P<$ 0.05 and $P<0.01$, respectively). However, we conclude that mMGL minimally mediates the loss of CD8 T cells in ST3Gal$I^{-/-}$mice. Either the loss of CD8 T cells in ST3Gal-I ${ }^{-1-}$ mice occurs by a different mechanism entirely, or there is another endogenous galactose-specific lectin that is functionally redundant with $\mathrm{mMGL}$ in this regard.

Evidence for multiple Galß1-3GalNAc lectins expressed on macrophages. As a probe to examine Gal/GalNAc lectins within lymphoid tissues, we used a biotinylated mucin known as AFGP, a highly glycosylated polypeptide containing multiple O-linked Galß1-3GalNAc disaccharides. As shown in Fig. 7A and B, AFGP bound to cells located in the medulla of the thymus (compared with the streptavidin control). To show the specificity of this binding, serial sections of the thymus were stained with AFGP-biotin and competed with Galß1-3GalNAc or Gal 1-3GalNAc. As shown in Fig. 7C-E, there was a specific competition with Gal $\beta 1-3 \mathrm{GalNAc}$ compared with Gal $\alpha 1-3 \mathrm{Gal}-$ NAc. Similar staining results in the thymus were found with a biotinylated polyacrylamide-based polymer of Gal 1 1-3GalNAc (Galß1-3GalNAc-PAA) (data not shown).

To determine whether the lectin was present on macrophages, spleen sections were stained with biotinylated Gal $\beta 1$ 3GalNAc-PAA or biotinylated anti-Mac-1, followed by alkaline phosphatase-labeled streptavidin (Fig. 8A). Similar to anti-mMGL, staining with the Gal/GalNAc polymer was sparse but definitely positive in the spleen compared with anti-Mac-1. To determine whether this staining was dependent on mMGL, sections from an $\mathrm{mMGL}^{-1-}$ mouse were also double-stained with anti-Mac-1-FITC and Gal/GalNAc-PAA-rhodamine (Fig. 8B). The pictures were taken separately and then merged. The inset shows the AFGP labeling alone. As shown, there was strong Gal/GalNAc probe binding despite the absence of mMGL, and all of the Gal/GalNAc staining coincided with Mac-1 staining. This indicates that the lectin detected by AFGP or Gal 1 1-3GalNAc-PAA is present on a small subpopulation of Mac-1-positive cells. Similar to mMGL itself, these cells could be a subpopulation of macrophages or dendritic cells. Preliminary evidence showed that this lectin-carbohydrate interaction may not be calcium dependent, as binding occurred in the presence of EDTA. Thus, at least one putative Gal/GalNAc binding molecule is most likely not a C-type lectin.

\section{DISCUSSION}

In this report we have described the production and characterization of mice deficient in the known macrophage galactose lectin mMGL. With these mice, we addressed three questions related to a potential role for $\mathrm{mMGL}$ in the recognition of cell surface structures regulated by differential sialylation. First, does mMGL regulate the maturation and progression of undersialylated, cortical, $\mathrm{CD} 4^{+} \mathrm{CD}^{+}$thymocytes? Second, does $\mathrm{mMGL}$ regulate the turnover of effector $\mathrm{T}$ cells that become undersialylated subsequent to activation? Third, does 

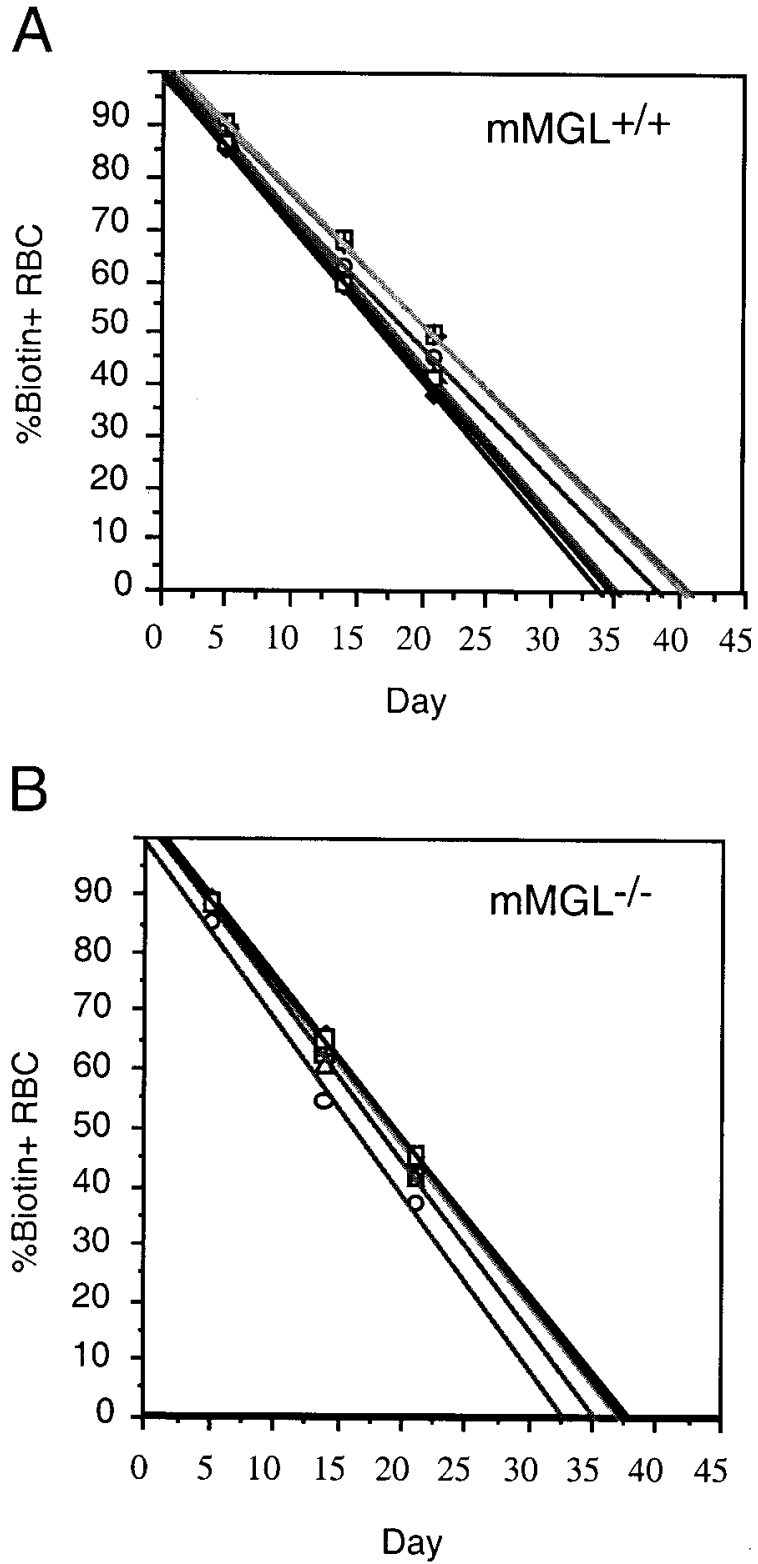

FIG. 5. Erythrocyte (RBC) turnover in $\mathrm{mMGL}^{+/+}$and $\mathrm{mMGL}^{-/-}$ mice. Mean life span of red blood cells was determined in (A) $\mathrm{mMGL}^{+/+}$and (B) $\mathrm{mMGL}^{-/-}$mice after biotinylation in vivo. The mean erythrocyte life span was 36.67 days in $m M G L^{+/+}$mice, versus 37.27 days in $m M G L^{-1-}$ mice.

mMGL play a role in the turnover of red blood cells that lose sialylation with age?

The development and selection of thymocytes are accompanied by a high rate of cell death, so that an estimated $98 \%$ of the thymocytes produced in the thymus die prior to emigration (31). The physiological mechanisms responsible for thymocyte cell death are almost entirely uncharacterized (37). Several receptor-ligand interactions have been proposed to participate in thymocyte death $(6,16,17,22)$, but there are few examples of targeted deletions that result in thymus hyperplasia (33). Despite the many studies on the topic, there is yet no real conceptual understanding of the signaling mechanisms that
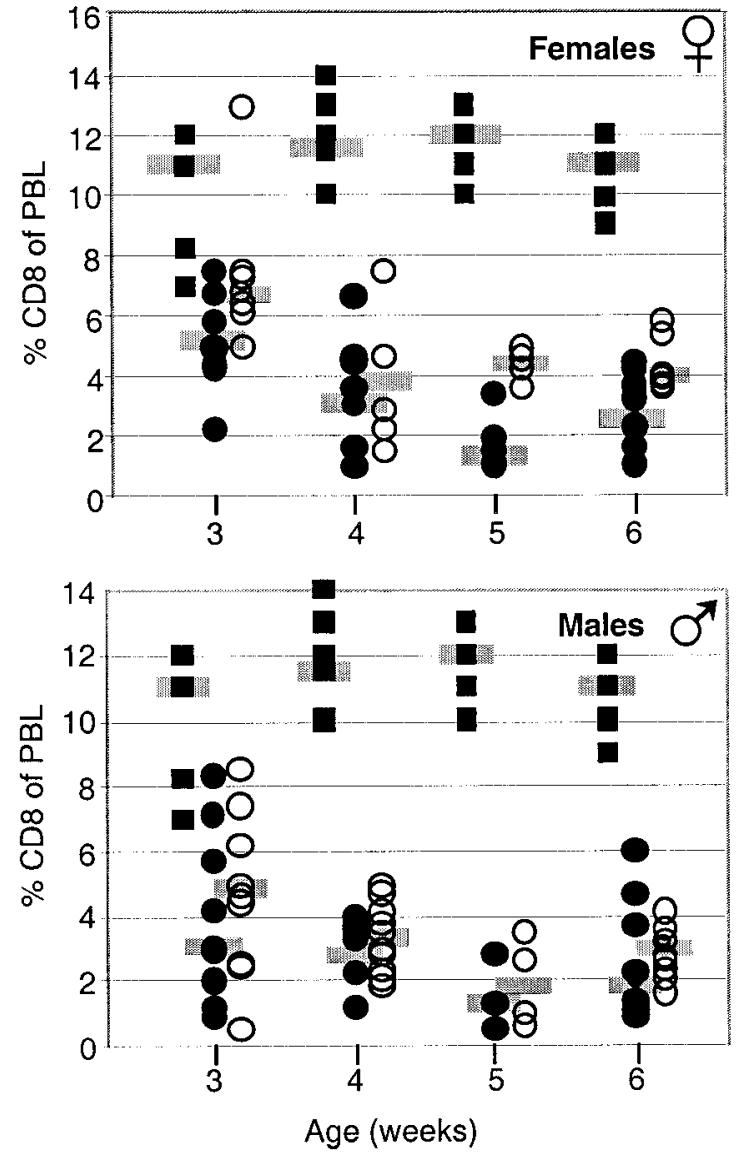

FIG. 6. Effect of mMGL deficiency in ST3Gal-I-deficient mice. $S T 3 G a l-I$ mice were crossed to $m M G L$-deficient mice to examine the role of mMGL in the CD8 T-cell loss in ST3Gal-I-deficient mice. Solid squares represent $S T 3 \mathrm{Gal}_{-} \mathrm{I}^{+/-}$mice, which show normal CD8 T-cell numbers in the peripheral blood lymphocytes (PBL). Solid circles

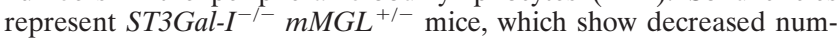
bers of CD8 $\mathrm{T}$ cells. Open circles show doubly deficient mice. (Top) Female doubly deficient mice show a trend of increased CD8 T-cell numbers compared to ST3Gal-I-deficient mice, reaching statistical significance in female mice aged 3 and 5 weeks. (Bottom) Male mice showed no statistically significant changes.

distinguish positive and negative selection of developing thymocytes. As such, we wanted to study the possibility that differential sialylation during thymus development could be recognized by an endogenous lectin as a way of regulating the emergence of mature T cells. From this and previous studies, we conclude that neither the deletion of ST3Gal-I nor the deletion of mMGL appears to have a substantial effect on T-cell development (23).

The observation that activated $\mathrm{T}$ cells lose sialylation and following expansion show significant apoptosis prompted us to consider the possibility that an endogenous lectin could induce death based on cross-linking of undersialylated molecules. This mechanism would be consistent with the loss of CD8 T cells in ST3Gal-I-deficient mice and the delay in death of antigenspecific effector CD8 T cells in CD43 ${ }^{-1-}$ mice (Onami et al., unpublished data). We saw no differences in CD8 T cell numbers for $m M G L^{+/+}$versus $m M G L^{-/-}$mice following activation in vivo and similar numbers of apoptotic CD8 T cells in 

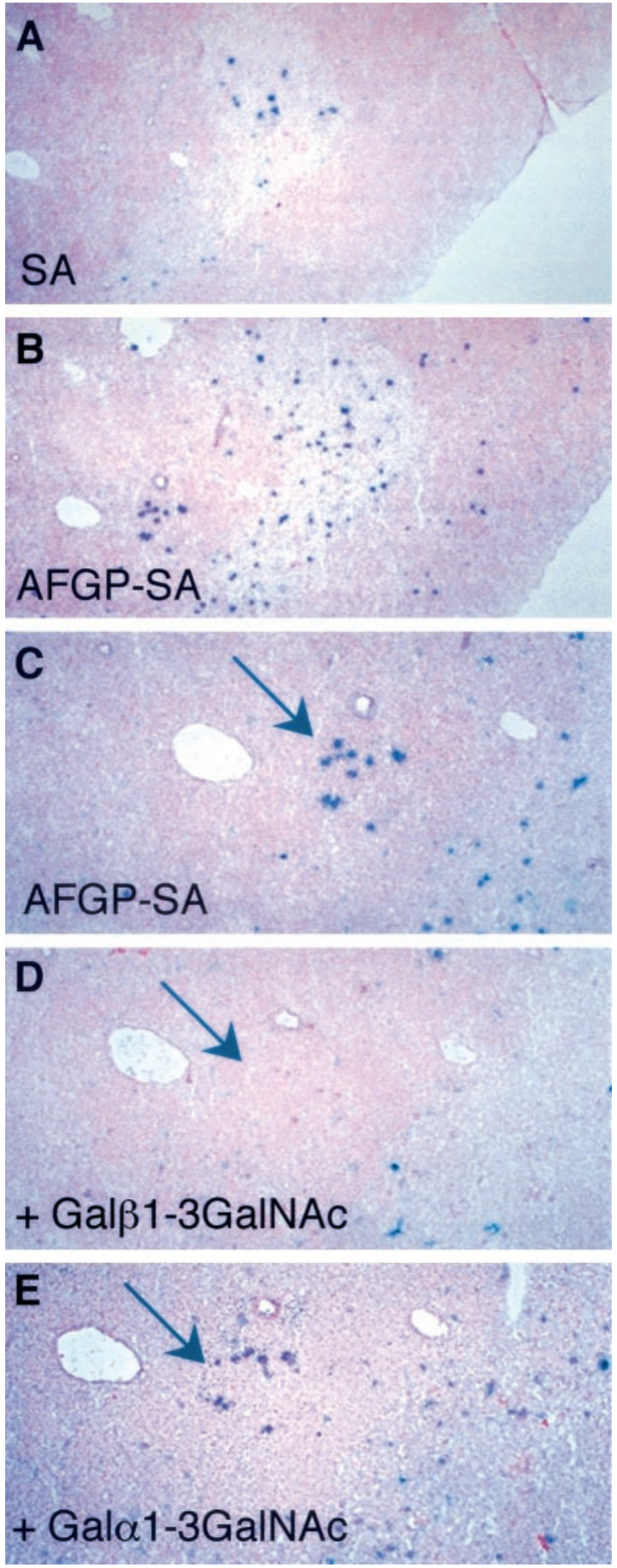

FIG. 7. Identification of Gal/GalNAc-specific lectin(s) distinct from mMGL. (A) Sialic acid (SA) binding alone. (B) GalB1-3GalNAcPAA. (C to E) Serial sections were probed with (C) Gal $\beta 1-3$ GalNAcPAA, (D) Galß1-3GalNAc-PAA plus Galß1-3GalNAc, and

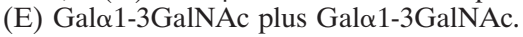

these mice as well. To determine whether an $m M G L$ gene inhibition could complement the targeted ST3Gal-I deficiency in the loss of CD8 T cells, we produced mice deficient in both genes. As shown, although there appeared to be a tendency toward higher numbers of $\mathrm{CD} 8 \mathrm{~T}$ cells in the mice lacking $m M G L, m M G L^{-1-} S T 3 G a l-I^{-/-}$mice still showed a profound
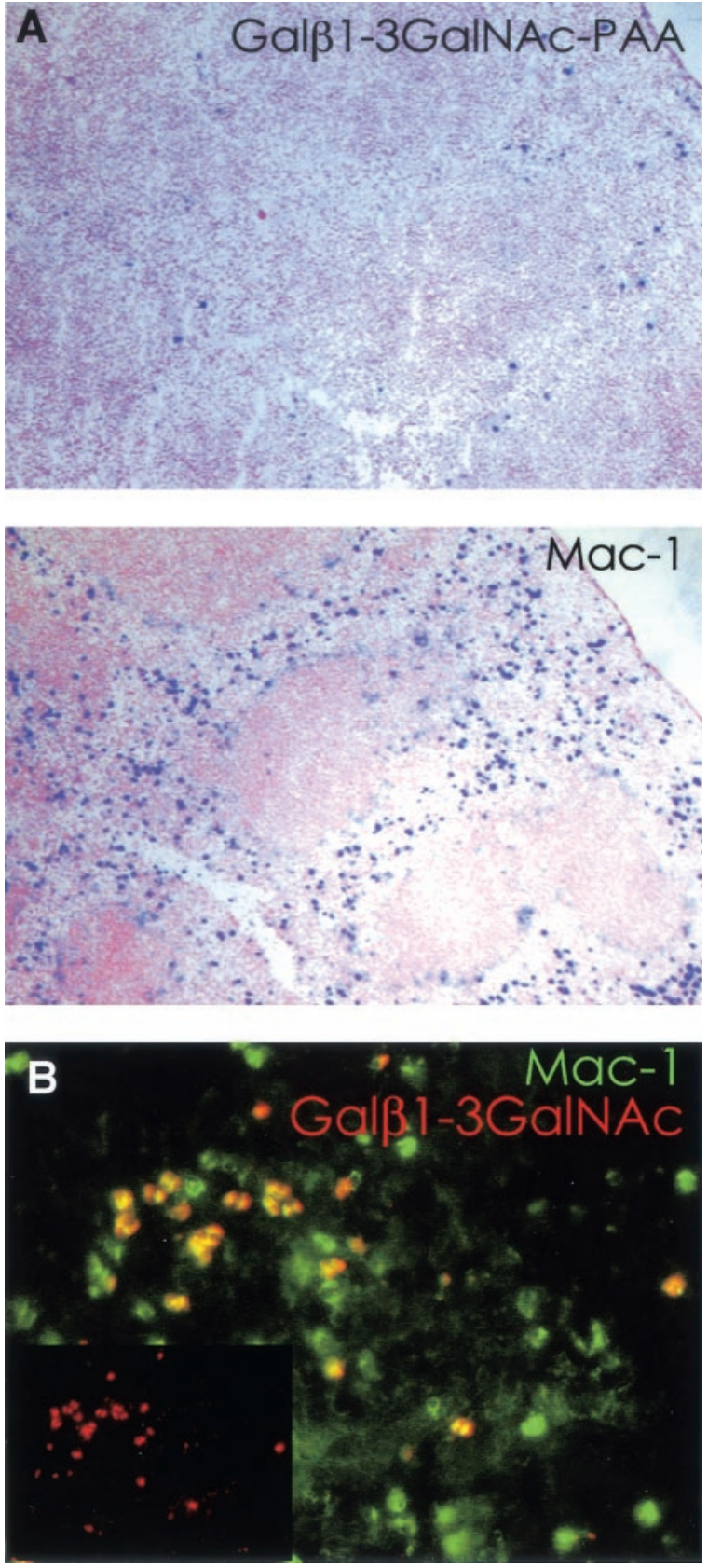

FIG. 8. Gal/GalNAc-specific lectin(s) is macrophage restricted. (A) Splenic sections from $m M G L^{-/-}$mice were probed with biotinylated GalB1-3GalNAc-PAA or anti-Mac-1 antibodies. Localized binding of the Galß1-3GalNAc-PAA probe was observed in the splenic red pulp, and this correlated with Mac-1 staining. (B) Double staining of splenic sections from $m M G L$-deficient mice with Galß1-3GalNAcPAA and Mac-1 revealed colocalization of the lectin to a Mac- $1^{+}$cell. Gal 1 1-3GalNAc-PAA binding was always seen on Mac-1 binding cells but not the converse, suggesting that the lectin(s) is expressed on a subpopulation of macrophages or mature dendritic cells.

CD8 T-cell deficiency. Therefore, we conclude that mMGL cross-linking of undersialylated cell surface molecules is not

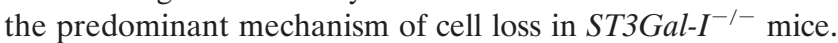

In addition to lymphocytes, erythrocytes undergo differential sialylation. Experiments show that sialic acids are lost as eryth- 
rocytes undergo senescence $(2,4,14)$. The role of sialic acids in erythrocyte senescence is not understood, but one possibility is that it constitutes one of the signals that target aged erythrocytes for destruction (9). We considered the possibility that mMGL-bearing macrophages could recognize the undersialylated erythrocytes and execute their engulfment. There does appear to be a difference in erythrocyte numbers in mMGL mice (Table 1), but the biological significance is difficult to discern. We did not find a statistically significant difference in turnover rates, leading us to believe that the increases seen in erythrocytes in $m M G L^{-1-}$ mice may be explained by other indirect effects. Moreover, mMGL expression is not abundant in the spleen and liver, where erythrocyte turnover takes place after desialylation.

With AFGP as a probe, we identified an additional macrophage lectin(s) or lectin-like activity that is specific for Galß13GalNAc but independent of mMGL. Binding of this lectin to its ligand is not affected by EDTA, suggesting that binding is not calcium dependent, and thus it is not a C-type lectin. In future studies, this lectin can be characterized further by purifying galactose-specific binding proteins with cell lysates derived from mMGL null mice. We propose that this lectin may play a functionally redundant role to mMGL and conclude from our studies that mMGL expression is not required for normal lymphoid homeostasis.

\section{ACKNOWLEDGMENTS}

This work was supported by Public Health Service grants RO1AI37988 (S.M.H.) and PO1HL57345 (A.V.) from the National Institutes of Health and the Howard Hughes Medical Institute (J.D.M.).

\section{REFERENCES}

1. Ault, K. A., and C. Knowles. 1995. In vivo biotinylation demonstrates that reticulated platelets are the youngest platelets in circulation. Exp. Hematol. 23:996-1001.

2. Balduini, C. L., F. Sinigaglia, E. Ascari, and C. Balduini. 1978. Behaviour of young and old desialylated rabbit erythrocytes in vivo. Hoppe Seyler's Z. Physiol. Chem. 359:1573-1577.

3. Baum, L. G., K. Derbin, N. L. Perillo, T. Wu, M. Pang, and C. Uittenbogaart. 1996. Characterization of terminal sialic acid linkages on human thymocytes. Correlation between lectin-binding phenotype and sialyltransferase expression. J. Biol. Chem. 271:10793-10799.

4. Bocci, V. 1981. Determinants of erythrocyte ageing: a reappraisal. Br. J. Haematol. 48:515-522.

5. Dumont, F., and J. Nardelli. 1979. Peanut agglutinin (PNA)-binding properties of murine thymocyte subpopulation. Immunology 37:217-224.

6. Foy, T. M., D. M. Page, T. J. Waldschmidt, A. Scheneveld, S. M. Hedrick, and R. Noelle. 1995. An essential role for gp39, the ligand for CD40, in thymic selection. J. Exp. Med. 182:1377-1388.

7. Fukuda, M. 1991. Leukosialin, a major O-glycan-containing sialoglycoprotein defining leukocyte differentiation and malignancy. Glycobiology 1:347356.

8. Galvan, M., K. Murali-Krishna, L. L. Ming, L. Baum, and R. Ahmed. 1998. Alterations in cell surface carbohydrates on T cells from virally infected mice can distinguish effector/memory CD8 + T cells from naive cells. J. Immunol. 161:641-648.

9. Gattegno, L., F. Fabia, D. Bladier, and P. Cornillot. 1979. Physiological ageing of red blood cells and changes in membrane carbohydrates. Biomedicine 30:194-199.

10. Gillespie, W., J. C. Paulson, S. Kelm, M. Pang, and L. G. Baum. 1993. Regulation of alpha 2,3-sialyltransferase expression correlates with conversion of peanut agglutinin (PNA) + to PNA - phenotype in developing thymocytes. J. Biol. Chem. 268:3801-3804.

11. Ichii, S., Y. Imai, and T. Irimura. 1997. Tumor site-selective localization of an adoptively transferred $\mathrm{T}$ cell line expressing a macrophage lectin. J. Leukoc. Biol. 62:761-770.

12. Ii, M., H. Kurata, N. Itoh, I. Yamashina, and T. Kawasaki. 1990. Molecular cloning and sequence analysis of cDNA encoding the macrophage lectin specific for galactose and N-acetylgalactosamine. J. Biol. Chem. 265:1129511298.
13. Imai, Y., Y. Akimoto, S. Mizuochi, T. Kimura, H. Hirano, and T. Irimura. 1995. Restricted expression of galactose/ $\mathrm{N}$-acetylgalactosamine-specific macrophage C-type lectin to connective tissue and to metastatic lesions in mouse lung. Immunology 86:591-598.

14. Jakubowska-Solarska, B., and J. Solski. 2000. Sialic acids of young and old red blood cells in healthy subjects. Med. Sci. Monit. 6:871-884.

15. Kawakami, K., K. Yamamoto, S. Toyoshima, T. Osawa, and T. Irimura. 1994. Dual function of macrophage galactose/N-acetylgalactosamine-specific lectins: glycoprotein uptake and tumoricidal cellular recognition. Jpn. J. Cancer Res. 85:744-749.

16. Kishimoto, H., and J. Sprent. 2000. The thymus and negative selection. Immunol. Res. 21:315-323.

17. Li, R., and D. M. Page. 2001. Requirement for a complex array of costimulators in the negative selection of autoreactive thymocytes in vivo. J. Immunol. 166:6050-6606.

18. London, J., S. Berrih, and J. F. Bach. 1978. Peanut agglutinin. I. A new tool for studying T lymphocyte subpopulations. J. Immunol. 121:438-443.

19. London, J., and M. A. Horton. 1980. Peanut agglutinin. V. Thymocyte subpopulations in the mouse studied with peanut agglutinin and Ly-6.2 antiserum. J. Immunol. 124:1803-1807.

20. Mizuochi, S., Y. Akimoto, Y. Imai, H. Hirano, and T. Irimura. 1997. Unique tissue distribution of a mouse macrophage C-type lectin. Glycobiology 7:137-146.

21. Page, D. M., L. P. Kane, J. P. Allison, and S. M. Hedrick. 1993. Two signals are required for negative selection of CD4+CD8+ thymocytes. J. Immunol. 151:1868-1880

22. Perillo, N. L., C. H. Uittenbogaart, J. T. Nguyen, and L. G. Baum. 1997. Galectin-1, an endogenous lectin produced by thymic epithelial cells, induces apoptosis of human thymocytes. J. Exp. Med. 185:1851-1858.

23. Priatel, J. J., D. Chui, N. Hiraoka, C. J. Simmons, K. B. Richardson, D. M. Page, M. Fukuda, N. M. Varki, and J. D. Marth. 2000. The ST3Gal-I sialyltransferase controls CD8 + T lymphocyte homeostasis by modulating O-glycan biosynthesis. Immunity 12:273-283.

24. Reed, D. S., S. Olson, and L. Lefrancois. 1998. Glycosyltransferase regulation mediated by pre-TCR signaling in early thymocyte development. Int. Immunol. 10:445-451.

25. Reisner, Y., M. Linker-Israeli, and N. Sharon. 1976. Separation of mouse thymocytes into two subpopulations by the use of peanut agglutinin. Cell. Immunol. 25:129-134.

26. Rose, M. L., M. S. Birbeck, V. J. Wallis, J. A. Forrester, and A. J. Davies. 1980. Peanut lectin binding properties of germinal centres of mouse lymphoid tissue. Nature 284:364-366.

27. Rose, M. L., and F. Malchiodi. 1981. Binding of peanut lectin to thymic cortex and germinal centres of lymphoid tissue. Immunology 42:583-591.

28. Russell, M. E., U. Utans, A. F. Wallace, P. Liang, R. J. Arceci, M. J. Karnovsky, L. R. Wyner, Y. Yamashita, and C. Tarn. 1994. Identification and upregulation of galactose/ $\mathrm{N}$-acetylgalactosamine macrophage lectin in rat cardiac allografts with arteriosclerosis. J. Clin. Investig. 94:722-730.

29. Sato, M., K. Kawakami, T. Osawa, and S. Toyoshima. 1992. Molecular cloning and expression of cDNA encoding a galactose/N-acetylgalactosamine-specific lectin on mouse tumoricidal macrophages. J. Biochem. (Tokyo) 111:331-336.

30. Schrader, J. W., W. F. Chen, and R. Scollay. 1982. The acquisition of receptors for peanut agglutinin by peanut agglutinin-negative thymocytes and peripheral T cells. J. Immunol. 129:545-549.

31. Shortman, K., and R. Scollay. 1994. Immunology. Death in the thymus. Nature 372:44-45.

32. Soriano, P., C. Montgomery, R. Geske, and A. Bradley. 1991. Targeted disruption of the c-src proto-oncogene leads to osteopetrosis in mice. Cell 64:693-702.

33. Suzuki, A., M. T. Yamaguchi, T. Ohteki, T. Sasaki, T. Kaisho, Y. Kimura, R. Yoshida, A. Wakeham, T. Higuchi, M. Fukumoto, T. Tsubata, P. S. Ohashi, S. Koyasu, J. M. Penninger, T. Nakano, and T. W. Mak. 2001. T cell-specific loss of Pten leads to defects in central and peripheral tolerance. Immunity 14:523-534.

34. Tannert, C., G. Schmidt, D. Klatt, and S. M. Rapoport. 1977. Mechanism of senescence of red blood cells. Acta Biol. Med. Ger. 36:831-886.

35. Tsuji, S., A. K. Datta, and J. C. Paulson. 1996. Systematic nomenclature for sialyltransferases. Glycobiology 6:v-vii.

36. Whiteheart, S. W., J. C. McLenithan, and G. W. Hart. 1990. Surfaces of murine lymphocyte subsets differ in sialylation states and antigen distribution of a major N-linked penultimate saccharide structure. Cell. Immunol. 125: 337-353.

37. Williams, O., and H. J. Brady. 2001. The role of molecules that mediate apoptosis in T-cell selection. Trends Immunol. 22:107-111.

38. Wu, W., J. A. Punt, L. Granger, S. O. Sharrow, and K. P. Kearse. 1997. Developmentally regulated expression of peanut agglutinin (PNA)- specific glycans on murine thymocytes. Glycobiology 7:349-356.

39. Yamamoto, K., C. Ishida, Y. Shinohara, Y. Hasegawa, Y. Konami, T. Osawa, and T. Irimura. 1994. Interaction of immobilized recombinant mouse C-type macrophage lectin with glycopeptides and oligosaccharides. Biochemistry 33:8159-8166. 